\title{
ANESTESIA PARA MIELOGRAFIA EM CÃES
}

\author{
ANESTHESIA FOR MYELOGRAPHY IN DOGS
}

\author{
Alessandra Valeiro Leite ${ }^{1}$ Newton Nunes ${ }^{2}$ Márlis Langenegger de Rezende ${ }^{3}$
}

\section{- REVISÃO BIBLIOGRÁFICA -}

RESUMO

\begin{abstract}
Com este texto, objetiva-se fornecer ao profissional de Medicina Veterinária subsídios para a realização de mielografia para o qual é necessário submeter o animal À anestesia geral. Este exame não é um procedimento inócuo, pois várias reações adversas podem ocorrer durante e após sua realização. $O$ conhecimento da farmacodinâmica dos agentes anestésicos é de fundamental importância para o Médico Veterinário escolher associações que proporcionem segurança e minimizem as complicações advindas da técnica. Uma série de cuidados no período pré-anestésico, a escolha de associações de fármacos adequada e o monitoramento do animal durante e após a mielografia proporcionarão aos pacientes uma maior segurança no procedimento.
\end{abstract}

Palavras-chave: neuroradiologia, convulsões, pressão intracraniana, protocolos anestésicos.

\section{SUMMARY}

The aim of this work was to supply the Veterinarian professional with information regarding to the execution of a mielography, which requires general anesthesia. This exam is not harmless and several adverse reactions may occur during and after its realization. The knowledge of the anesthetics pharmacodynamic is essential in order to select the associations that could provide safety and minimize the myelography complications. Some procedures in the preanesthetic period, the selection of the appropriate anesthetic agents and monitoring the animal during and after the myelography will provide major safety to this exam.

Key words: neuroradiology, seizures, intracranial pressure, anesthetics protocols.

\section{INTRODUÇÃO}

A injeção de contrastes radiopacos no espaço subaracnóide é a base de uma técnica neurorradiográfica conhecida como mielografia. Através dessas imagens, é possível detectar compressões medulares responsáveis por alterações neurossistêmicas. Para a realização desse exame, é necessária a anestesia geral por via intravenosa ou inalatória, monitoração constante e suporte ventilatório adequado.

Levando em consideração os riscos inerentes à técnica radiográfica da mielografia e a necessidade de fármacos que proporcionem maior segurança para o procedimento, a presente revisão propõe tecer considerações a respeito de fármacos anestésicos e protocolos possíveis para a realização deste exame e dos cuidados clínicos a serem tomados antes e após a anestesia.

\section{CONSIDERAÇÕES GERAIS}

A primeira mielografia, no homem, foi realizada por volta de 1920 utilizando-se, como meio de contraste, o ar e o oxigênio (BULLOCK \& ZOOK, 1967). Apesar destes meios produzirem um contraste negativo, não forneciam bons diagnósticos pois não se misturavam ao líquido cérebro espinhal (LCE) levando a falhas no delineamento da medula espinhal (SARMENTO et al., 2000). Em 1931, foi introduzido, na Europa, o uso do methiodal sódico,

\footnotetext{
${ }^{1}$ Pós-graduanda do Curso de Mestrado em Cirurgia Veterinária, Área de concentração em Cirurgia, Faculdade de Ciências Agrárias Veterinárias (FCAV) Universidade Estadual Paulista (UNESP), Campus de Jaboticabal, SP.

${ }^{2}$ Professor Assistente, Doutor Departamento de Clínica e Cirurgia Veterinária, FCAV, UNESP, Campus de Jaboticabal, 14870-000, Jaboticabal, SP. E-mail: newton@fcav.unesp.br. Autor para correspondência.

${ }^{3}$ Pós-Graduando do Curso de Doutorado em Medicina Veterinária, Área de Concentração em Cirurgia, FCAV, UNESP. 
um meio hidrossolúvel, iodado, de melhor qualidade radiográfica, mas tóxico para o tecido nervoso, gerando convulsões e agravamento dos sinais neurológicos. Uma década mais tarde surgiram os contrastes oleosos, menos tóxicos que os anteriores, mas que tendiam a formar globulações prejudicando a interpretação da imagem radiográfica e dificultando a sua remoção do espaço subaracnóide (BULLOCK \& ZOOK, 1967).

Em 1950, foi relatada a primeira mielografia realizada em cães (PUGLISI et al., 1986) e, embora já existissem alguns meios de contrastes oleosos disponíveis, nenhum era livre de causar reações adversas nos pacientes, pois a atração destes contrastes pelas camadas bilaminares das membranas celulares neurais causavam leptomeningites e outras reações neurológicas (SARMENTO et al., 2000).

O primeiro contraste iodado não iônico, utilizado no homem e em animais, foi o metrizamide (WIDMER, 1989; VANBREE et al., 1991), que, apesar da boa qualidade radiográfica, foi associado a altos índices de reações adversas.

Os meios de contraste iopamidol e iohexol pertencem à segunda geração de compostos iodados não iônicos solúveis em água e são os mais utilizados em Medicina Veterinária (WIDMER, 1989; HOLLAND, 1993). Eles podem ser autoclavados diminuindo, assim, o seu custo por aproveitamento de resíduos (SARMENTO et al., 2000), entretanto a menor neurotoxicidade não os isenta de causar reações adversas (VANBREE $\boldsymbol{e t} \boldsymbol{a l}$., 1991).

Dentre as complicações relatadas em mielografias, estão a apnéia durante a punção e aplicação do contraste, bradicardia, convulsões parciais ou generalizadas, exacerbação de sinais de disfunção do sistema nervoso central (SNC), vômitos, hipertemia, retenção urinária, mais raramente meningite asséptica e morte (BARTELS et al.,1978; PUGLISI et al., 1986; WIDMER, 1989; LEWIS \& HOSGOOD, 1992). As convulsões representam $75 \%$ das complicações ocorridas (TICER, 1987), principalmente quando as aplicações de contraste são feitas através da cisterna magna (WIDMER, 1989) e quando o animal apresenta espondilomielopatia cervical caudal (LEWIS \& HOSGOOD, 1992). Também a velocidade muito rápida de aplicação do contraste pode fazer com que esse flua para o espaço subaracnóide rostral acumulando-se no quarto ventrículo e no hemisfério cerebelar, aumentando a pressão intracraniana (PIC) e agravando as anormalidades neurológicas (BARTELS et al., 1978; WIDMER, 1989).
Outros fatores podem influenciar a prevalência de convulsões pós-mielográficas, entre eles, o protocolo anestésico, a duração da anestesia, a administração de fluidos intravenosos durante e após o procedimento, o tipo e a concentração do contraste (WIDMER, 1989; LEWIS \& HOSGOOD, 1992). Concentrações hipertônicas provocam um desequilíbrio iônico neuronal e a presença de 2deoxy-D-glucose na molécula de alguns contrastes, como o metrizamide, interfere no transporte de glicose intracelular feito através da membrana celular, desequilibrando o metabolismo neural (PUGLISI et al., 1986; WIDMER, 1989; LEWIS \& HOSGOOD, 1992).

O meio de contraste não se acumula dentro das células do SNC. Sua eliminação é um processo passivo e ocorre dentro do fluxo normal do LCE para o sistema venoso. Assim sendo, a desidratação diminui a liberação do meio de contraste do espaço subaracnóide acentuando os efeitos do fármaco no SNC. Quarenta e oito horas após a administração intratecal o contraste ainda é metabolizado pelo rim (WIDMER, 1989).

Várias combinações anestésicas são relatadas para o procedimento. Os fármacos préanestésicos mais utilizados são a atropina, glicopirrolato, meperidina, acepromazina, oximorfona, butorfanol e diazepam. A indução com barbitúricos e a manutenção com os anestésicos voláteis halotano ou isoflurano, associados ou não com óxido nitroso, são rotineiramente utilizadas (LEWIS \& HOSGOOD, 1992). Entretanto existem fármacos introduzidos na rotina em grandes centros hospitalares, como o desflurano e o sevoflurano, porém são desconhecidas as inter-relações entre esses fármacos e os contrastes de uso rotineiro para o exame.

\section{CUIDADOS PRÉ-ANESTÉSICOS}

A anestesia geral é imprescindível para a realização da mielografia, portanto, devem ser verificados antecedentes convulsivos, reações anteriores a drogas anestésicas, além do estado geral do paciente. Para uma melhor avaliação da função cardíaca, os animais idosos necessitam ser submetidos ao eletrocardiograma e à radiografia torácica. Previamente à anestesia, os animais precisam se submetidos a jejum hídrico de seis horas e alimentar de doze horas.

O exame neurológico avaliará o grau do status mental, marcha, função de nervos cranianos, reação postural e reflexos de segmentos espinhais (VANBREE et al., 1991). Essa análise detalhada da 
função do SNC é importante para a posterior avaliação do agravamento de sinais de disfunções do SNC já existentes.

Os desequilíbrios hidroeletrolíticos devem ser corrigidos antes da mielografia. Os animais suspeitos de terem a PIC aumentada ou em estado de choque após acidente traumático devem ser previamente estabilizados. Pacientes com processos infecciosos sistêmicos ou com infecção cutânea no local da injeção não devem ser submetidos ao exame pois a agulha pode contaminar o líquor $\mathrm{e}$, conseqüentemente, as meninges e medula (SIMON \& NICHOLAS, 1999).

\section{ESCOLHA DOS FÁRMACOS E DAS TÉCNICAS}

Independentemente de se utilizar anestesia volátil, todos os animais submetidos ao exame de mielografia necessitam estar intubados e durante todo o procedimento deve estar disponível um suporte de oxigênio (GREENE et al., 1999).

Para a medicação pré-anestésica, deve-se evitar o uso de fenotiazínicos, pois estes potencializam atividades convulsivantes (McKELVEY \& HOLLINGSHEAD, 2000). Os $\alpha 2$ agonistas também devem ser evitados pois o seu uso está associado a alguns efeitos indesejáveis como bradicardia, bloqueios cardíacos, sensibilização do coração aos efeitos arritmogênicos da adrenalina, depressão respiratória dose-dependente, que pode evoluir para severa hipoventilação com hipercarbia e o aumento da pressão no LCE (GREENE et al., 1999).

Os benzodiazepínicos exercem mínimo efeito sobre os sistemas cardiovascular e respiratório (McKELVEY \& HOLLINGSHEAD, 2000) além de possuirem um marcante potencial anticonvulsivante. São indicados na medicação pré-anestésica para coleta de LCE e mielografias, associados com pequenas doses de opióides (GREENE et al., 1999). Os opióides podem ser utilizados em pacientes com a PIC aumentada. Seus efeitos diretos no fluxo sangüíneo cerebral (FSC) são mínimos, entretanto podem indiretamente aumentar a pressão no LCE devido ao acúmulo de dióxido de carbono $\left(\mathrm{CO}_{2}\right)$ resultante da hipoventilação; devem ser utilizados em baixas doses e com cautela em pacientes com traumatismo e tumores cerebrais (GREENE et al., 1999).

Os agentes dissociativos, como a cetamina e a tiletamina, não são indicados para o procedimento devido ao aumento do FSC, da PIC e da pressão no LCE que ocorrem como consequência da vasodilatação cerebral e do aumento da pressão arterial (LIN, 1999). Pode ocorrer hipertonicidade, tremores musculares e aumento dos riscos de convulsão durante a recuperação anestésica (McKELVEY \& HOLLINGSHEAD, 2000).

Os barbitúricos são bastante utilizados na indução e manutenção anestésica em mielografias. Eles provocam uma depressão direta do centro bulbar, ocasionando a diminuição da amplitude e da freqüência respiratória, da freqüência cardíaca e da temperatura (MASSONE, 1999). Também promovem a redução da taxa de metabolismo cerebral, do FSC e da PIC (GREENE et al., 1999). O tiopental, um barbitúrico de ultracurta ação, tem sido incluido em diversos estudos mielográficos como agente indutor e na manutenção da anestesia. Os seus efeitos na redução da incidência de convulsões após mielografias ainda não são conclusivos; alguns autores acreditam que a diminuição na taxa da sua metabolização, quando associado com outras drogas também metabolizadas no fígado, pode resultar em concentrações remanescentes no sangue reduzindo o risco de convulsões (GRAY et al., 1987). Quando são administradas doses complementares, o fenômeno, denominado efeito cumulativo, pode estender o período de recuperação (MASSONE, 1999).

O propofol, um anestésico geral não barbitúrico, de uso exclusivo intravenoso, de ação rápida e de ultracurta duração, pode ser usado como agente de indução e também manutenção, através de infusão contínua ou em bolus (DENEUCHE \& DESBOIS, 1999). Depressão respiratória e apnéia são vistas, freqüentemente, após a sua administração, principalmente com injeções rápidas (MUIR \& GADAWSKI, 1998).

O fármaco apresenta efeitos dosedependentes e características semelhantes às do tiopental sódico, no entanto, o propofol não apresenta efeito cumulativo sendo distribuído de forma ampla e rapidamente eliminado, proporcionando recuperação anestésica mais rápida (McKELVEY \& HOLLINGSHEAD, 2000). Entretanto, alguns autores observaram maior tempo de recuperação da anestesia em cães submetidos a infusão contínua de propofol por tempos maiores que trinta minutos (ROBERTSON et al., 1992). Apresenta efeitos hemodinâmicos cerebrais semelhantes aos do tiopental, com redução do FSC, da PIC e do rítmo metabólico cerebral (LEMKE, 1999), podendo diminuir a pressão de perfusão cerebral, devido aos seus efeitos sobre a pressão arterial média. Possui também propriedades vasoconstritoras e atividades pró-convulsivantes e anticonvulsivante. Os efeitos pró-convulsivantes podem representar ativação de focos epileptogênicos. Os efeitos anticonvulsivantes são 
mais prováveis devido à depressão cortical inespecífica que pela ativação do limiar de convulsão (OMOIGUI, 1998).

Entre os anestésicos voláteis utilizados em Medicina Veterinária, o halotano bloqueia dramaticamente a autorregulação cerebral. Apesar de PUGLISI et al. (1986) afirmarem que o halotano não potencializa reações adversas durante ou após o exame de mielografia, o seu uso deve ser cauteloso. Estudos revelam um aumento de quase $200 \%$ no FSC em cães mantidos com 1,1 CAM de halotano e esse aumento pode causar herniação cerebral ou cerebelar em animais com a PIC já elevada. O aumento do FSC ocorre rapidamente após a administração do agente e independentemente de mudanças na pressão sangüínea arterial (GREENE et al., 1999).

O isoflurano causa pouca redução na função cardiovascular e o ritmo cardíaco permanece estável. Da mesma forma, tem pouca tendência a sensibilizar o miocárdio às catecolaminas, quando comparado com o halotano. Existem evidências constatando que ocorre redução dose-dependente na freqüência respiratória (McKELVEY \& HOLLINGSHEAD, 2000). Concentrações maiores que 1,1 CAM causam aumento no fluxo e no volume sangüíneo cerebral. A elevação na PIC é correspondente ao aumento no FSC; esse aumento é atenuado com o tempo e reflete o retorno da autorregulação vascular cerebral (OMOIGUI, 1998). A hiperventilação pulmonar é efetiva para evitar o aumento da PIC (NISHIYAMA, 1999).

O sevoflurano atua de maneira semelhante ao isoflurano. Sobre o sistema cardiovascular promove queda da pressão arterial média, deprime a contractilidade do miocárdio e não o sensibiliza à ação das catecolaminas (OMOIGUI, 1998). Comparado aos demais anestésicos voláteis, parece ser o que oferece maior estabilidade cardiovascular (EBERT et al., 1995).

A depressão respiratória produzida pelo agente anestésico ocorre de modo semelhante à causada pelo isoflurano, ou seja, diretamente sobre o centro ventilatório medular e indiretamente sobre os músculos intercostais (OMOIGUI, 1998). O FSC é preservado ou levemente diminuído. Com 1,1 CAM, o consumo de oxigênio cerebral pode ficar reduzido a $50 \%$ e a autorregulação do fluxo sangüíneo cerebral como resposta às mudanças do $\mathrm{PaCO}_{2}$ é, geralmente, preservada. Em condições de hipocapnia, o aumento dose dependente na PIC e a diminuição na resistência cerebrovascular podem não ser observadas (SCHWENDER et al., 1998).

Dentre os anestésicos voláteis, o desflurano é o fármaco mais recente introduzido para uso veterinário. A depressão respiratória pode ocorrer de modo semelhante à que se observa com o isoflurano e o sevoflurano (OMOIGUI, 1998). Promove redução dose-dependente da pressão arterial, resultante da diminuição da resistência vascular sistêmica (GRUNDMANN et $\boldsymbol{a l}$., 1996) e aumento da freqüência cardíaca (CLARKE et al., 1996) porém o débito cardíaco e a perfusão dos órgãos são mantidos (GRAHAM, 1994).

A vasodilatação cerebral produzida pelo desflurano causa aumento no fluxo e volume sangüíneos cerebrais. O aumento no fluxo sangüíneo cerebral é atenuado com o tempo e reflete retorno da autorregulação vascular cerebral independentemente da sua ação vascular sistêmica (BRENET et $\boldsymbol{a l}$., 1998). À semelhança do isoflurano, a reatividade do $\mathrm{CO}_{2}$ é preservada e a hiperventilação pulmonar pode diminuir o fluxo sangüíneo cerebral e a PIC (OMOIGUI, 1998).

A combinação de anestésicos voláteis com óxido nitroso causa um profundo aumento no FSC e PIC. Em coelhos, a administração de óxido nitroso, combinada com o halotano, isoflurano ou fentanil-pentobarbital proporcionou significantes aumentos no FSC e PIC sendo que a hiperventilação não minimiza esses efeitos adversos (GREENE et al., 1999).

\section{CUIDADOS PÓS-EXAME}

Após o término do exame, o animal deve permanecer sob monitoramento das funções vitais e com a cabeça elevada em 5 a $10^{\circ}$ para promover migração caudal do meio de contraste. $O$ paciente deve ser observado até estar consciente e ser capaz de manter a cabeça elevada (SIMON \& NICHOLAS, 1999). A fluidoterapia durante a recuperação auxilia na remoção do contraste do espaço subaracnóide (WIDMER, 1989).

A remoção do contraste imediatamente após mielografia, através da punção na cisterna magna, reduz a probabilidade de ocorrerem convulsões em cães, porém essa manobra só é indicada em pacientes cujo contraste fluiu para o espaço subaracnóide rostral (WIDMER, 1989).

O fármaco de eleição para o controle das convulsões é o diazepam $(1,0 \mathrm{mg} / \mathrm{kg})$ administrado por via intravenosa (McKELVEY \& HOLLINGSHEAD, 2000; SIMON \& NICHOLAS, 1999). É prudente deixar um cateter intravenoso fixado no paciente durante todo o período de recuperação possibilitando um acesso rápido para aplicações terapêuticas emergenciais.

A deterioração neurológica pode ocorrer após a prática da mielografia. Nesses casos, devem ser realizados novos exames neurológicos nas 24 horas que se seguem. Essa deterioração é geralmente transitória, e a maioria dos pacientes retorna ao estado prévio do mielograma em poucos dias (SIMON \& NICHOLAS, 1999). Se houve falhas na 
punção espinhal ou na técnica de injeção, pode resultar em significante lesão neurológica irreversível e até mesmo a morte.

\section{CONSIDERAÇÕES FINAIS}

Não há na literatura consultada um protocolo único e ideal para mielografias. É necessário o conhecimento da história clínica do paciente, o seu estado geral e o conhecimento das alterações hemodinâmicas proporcionadas pelos fármacos para permitir a escolha da associação mais segura para o paciente e que viabilize o procedimento.

\section{REFERÊNCIAS BIBLIOGRÁFICAS}

BARTELS, J.E., HOERLEIN, B.F., BORING, J.G. Canine neurology. Diagnosis and treatment. 3.ed. Philadelphia : Saunders, 1978. p.103-135.

BRENET, O, GRANRY, J.C., POIRIER, N., et al Effects of desflurane on cerebral blood flow velocities and cerebrovascular $\mathrm{CO}_{2}$ reactivity in children. Annales Francaises d Anesthesie et de Reanimation. v.17, n.3, p.227-233, 1998

BULLOCK, L.P., ZOOK, B.L. Myelography in dogs using water-soluble contrast mediums. Journal of the American Veterinary Medical Association, v.151, n.3, p.321-327, 1967.

CLARKE, K.W., ALIBHAI H.I.K., LEE Y.H., et al. Cardiopulmonary effects of desflurane in the dog during spontaneous and artificial ventilation. Research in Veterinary Science, , v.61, p.82-86, 1996.

DENEUCHE, A., DESBOIS, C. Propofoll - Pharmacological features. Point Veterinaire, v.30, n.201, p.29-34, 1999.

EBERT, T.J., HARKIN, C.P., MUZI, M. Cardiovascular responses to sevoflurane; a review. Anesthesia and Analgesia, v.81, n.6, p.11-22, 1995.

GRAHAM, S.G. New drug in volatile anaesthesia-desflurane. Annais of the Academy of Medicine Sigapore, v.23, n.4, p.510-518, 1994.

GRAY, P.R., INDRIERI, R.J., LIPPERT, A.C. et al. Influence of anesthetic regimen on the frequency of seizures after cervical myelography in the dog. Journal of the American Veterinary Medical Association, v.190, n.5, p.527-530, 1987.

GREENE, S.A., HARVEY, R.C., SIMS, M.H. Neurologic disease. In: THURMON, J.C., TRANQUILLI, W.J., BENSON, G.J. Essentials of small animal anesthesia \& analgesia. Lippincott : Williams \& Wilkins, 1999. p.437-447.

GRUNDMANN, U., MULLER, M., KLEINSCHMIDT, S. et al. Cardiovascular effects of desflurane and isoflurane in patients with coronary artery disease. Acta Anaesthesilogica Scandinavica, v.40, n.9, p.1101-1107, 1996

HOLLAND, M. Contrast agents. The Veterinary Clinics of North America: Small Animal Practice, v.23, n.2, p.269279, 1993.
LEMKE, K. Injectable anesthetics. In: THURMON, J.C., TRANQUILLI, W.J., BENSON, G.J. Essentials of small animal anesthesia \& analgesia. Lippincott : Williams \& Wilkins, 1999. p. 147.

LEWIS, D.D., HOSGOOD, G. Complications associated with the use of iohexol for myelography of the cervical vertebral column in dogs: 66 cases (1988-1990). Journal of the American Veterinary Medical Association, v.200, n.9, p.1381-1384, 1992.

LIN, H.C. Dissociative Anesthetics. In: THURMON, J.C., TRANQUILLI, W.J., BENSON, G.J. Essentials of small animal anesthesia \& analgesia. Lippincott : Williams \& Wilkins, 1999. p.151-153.

MASSONE, F. Anestesia veterinária. Farmacologia e técnicas. 3.ed. Rio de Janeiro : Guanabara Koogan, 1999. p.53-58.

McKELVEY, D., HOLLINGSHEAD, K.W. Small animal anesthesia \& analgesia. 2.ed. StLouis, 2000. 334p.

MUIR, W.W., GADAWSKI, J.E. Respiratory depression and apnea induced by propofol in dogs. American Journal of Veterinary Research, v.59, n.2, p.157-161, 1998.

NISHIYAMA, T., MATSUKAWA, T., YOKOYAMA, T. et al Cerebrovascular carbon dioxide reactivity during general anesthesia: A comparison between sevoflurane and isoflurane. Anesthesia and analgesia, v.89, n.6, p.14371441,1999

OMOIGUI, S. Manual de drogas usadas em anestesia. 2.ed. São Paulo : Santos, 1998. 566p.

PUGLISI, T.A., GREEN, R.W., HALL, C.L., et al. Comparison of metrizamide and iohexol for cisternal myelographyc examination of dogs. American Journal of Veterinary Research, v.47, n.8, p.1863-1869, 1986.

ROBERTSON, S.A., JOHNSTON, S., BEEMSTERBOER, J. Cardiopulmonary, anesthetic postanesthetic effects of intravenous infusions of propofol in greyhounds and nongreyhounds. American Journal of Veterinary Research, v.53, n.6, p.1027-1032, 1992.

SARMENTO, L.V.C., TUDURY,E.A., MAGALHÃES, P.K.L. $\boldsymbol{e} t$ al. Mielografia em cães. Clínica Veterinária, ano V,. n.26, p.23-32, 2000

SCHWENDER, D., END, H., DAUNDERER, M. et al. Sevoflurane and the nervous system. Anaesthesist, v.47, p.537-542, 1998.

SIMON, J.W., NICHOLAS, J.H.S. Diagnóstico e tratamento cirúrgico das afecções espinais do cão e do gato. São Paulo: Manole, 1999. p.46-48.

TICER, J.W. Radiographic technique in small animal practice. 2.ed. Pliladelphia : Saunders, 1987. p.218-224.

VANBREE, H., VANRIJSSEN,B., VANHAM, L. Comparison of nonionic contrast agents iohexol and iatrolan for cisternal myelography in dogs. American Journal of Veterinary Research, v.52, n.6, p.926-933, 1991.

WIDMER, W.R. Iohexol and iopamidol: new contrast media for veterinary myelography. Journal of the American Veterinary Medical Association, v.194, n.12, p.1714-1716, 1989. 